\title{
Ecological Evaluation of Port Resources Based on BP Neural Network
}

\author{
Jianxia Wan ${ }^{\text {a }}$,Shukui Zhang ${ }^{\mathrm{b}}$,Jing $\mathrm{Li}^{\mathrm{c}}$ \\ Navigational Department Jiangsu Maritime Institute, Nanjing 211170, Jiangsu,China. \\ a2091391569@qq.com,bzhangshkfy@163.com, c158142476@qq.com
}

\begin{abstract}
In order to improve the comprehensive evaluation method on ecological of port resources, a new general index system is established on the basis of researched results. An integrated evaluation model of ecology is built on the base of theory of BP network. Based on the ecology status of port resource, 10 samples are selected to train BP network and the training error reaches $1 \times 10^{-3}$. The ecological of 17 port resources are assessed with the evaluation model of BP network.
\end{abstract}

Keywords: ecological; evaluation; BP network; principal component analysis.

\section{Introduction}

The ecological quality of port resources covers water resources, land coastline resources, energy ecological resources as well as pollution accident prevention and response. Scientific evaluation of ecological quality is a prerequisite for port pollution control and safety management decisions. It is of great significance to improve port environmental pollution and improve the efficiency of port resource utilization.

At present, the commonly used evaluation methods include gray system theory, fuzzy comprehensive evaluation, principal component analysis, grey correlation analysis and RS-SPA evaluation, although these evaluation methods are more objective, in the evaluation process, the original index information will be lost, which will affect the accuracy of the evaluation results.

A BP neural network evaluation model is to be built in the paper to make up for the insufficiency of the above evaluation methods.

\section{Evaluation Index and Evaluation Sample}

\subsection{Evaluation Index}

The factors that affect the ecology of port resources have been researched in-depth and relevant research results are referenced in the paper, then the evaluation index system for ecological quality of port resources is summarized shown in Table 1.

Table.1 Evaluation index system for ecological quality of port resources

\begin{tabular}{|c|c|}
\hline Evaluation index & Index definition \\
\hline visibility(d/year)U1 & average days of visibility less than 1,000 meters per year \\
\hline Strong wind(d/year)U2 & Average days of winds above level 6 per year \\
\hline Maximum flow rate(m/s)U3 & Maximum flow rate of waterway \\
\hline waterway depth U4 & ratio of ship average draught to waterway depth \\
\hline Waterway width U5 & ratio of ship average width to waterway width \\
\hline Number of crossing points U6 & number of crossing and branch points \\
\hline Number of turning points U7 & Total length of waterway \\
\hline Waterway length (n mile)U8 & Average daily traffic volume \\
\hline Obstacle distribution U9 & Influence of obstructions distribution on the Safety of Navigation \\
\hline Weighted traffic (ships /d)U10 & waterway can pass through ship's tonnage \\
\hline Navigation standard (t)U11 & VTS management, service level \\
\hline VTS service U12 &
\end{tabular}


In the evaluation index, expert's scoring method was used for obstacles distribution and VTS service, experts assign 1, 2, 3, 4, 5 according to five security levels of excellent, good, medium, poor and worst, and the remaining 10 indicators are represented by numbers.

\subsection{Evaluation Sample}

According to the indicators for ecological quality evaluation of port resources, 17 domestic ports were selected for sample data collection, and collection methods include citations of existing research results, on-site research, questionnaires, online reviews, etc. Organized and summarized the collected data and results are shown in Table 2.

Table. 2 evaluation index data of each port

\begin{tabular}{|c|c|c|c|c|c|c|c|c|c|c|c|c|}
\hline $\begin{array}{c}\text { index } \\
\text { port }\end{array}$ & $\mathrm{U} 1$ & $\mathrm{U} 2$ & $\mathrm{U} 3$ & $\mathrm{U} 4$ & $\mathrm{U} 5$ & $\mathrm{U} 6$ & $\mathrm{U} 7$ & $\mathrm{U} 8$ & $\mathrm{U} 9$ & $\mathrm{U} 10$ & $\mathrm{U} 11$ & $\mathrm{U} 12$ \\
\hline DalianT1 & 26.9 & 40.2 & 3.0 & 0.67 & 0.15 & 5 & 3 & 9.5 & 2 & 54.54 & 5 & 2 \\
\hline QinhuangdaoT2 & 11.2 & 8.6 & 3.0 & 0.61 & 0.25 & 2 & 3 & 14.0 & 2 & 29.25 & 5 & 2 \\
\hline TianjinT3 & 9.9 & 80.0 & 1.5 & 0.64 & 0.21 & 1 & 1 & 20.4 & 2 & 40.22 & 7 & 1 \\
\hline YantaiT4 & 23.5 & 76.4 & 0.5 & 0.58 & 0.24 & 2 & 2 & 4.2 & 1 & 14.35 & 5 & 3 \\
\hline QingdaoT5 & 54.2 & 94.2 & 3.0 & 0.64 & 0.07 & 8 & 3 & 7.4 & 1 & 43.54 & 10 & 2 \\
\hline RizhaoT6 & 21.5 & 15.4 & 2.4 & 0.54 & 0.18 & 1 & 3 & 6.1 & 2 & 22.45 & 10 & 2 \\
\hline LlianyungangT7 & 16.2 & 138.3 & 2.9 & 0.75 & 0.34 & 3 & 4 & 3.6 & 2 & 14.01 & 7 & 3 \\
\hline ShanghaiT8 & 24.7 & 51.3 & 6.0 & 0.62 & 0.13 & 16 & 13 & 85.5 & 3 & 76.24 & 10 & 1 \\
\hline ZhangjiagangT9 & 26.2 & 48.3 & 6.2 & 0.67 & 0.35 & 4 & 7 & 4.5 & 2 & 38.52 & 5 & 2 \\
\hline BeilunT10 & 28.1 & 21.4 & 5.0 & 0.53 & 0.21 & 6 & 3 & 13.6 & 2 & 21.95 & 10 & 2 \\
\hline TaizhouT11 & 22.4 & 30.2 & 4.8 & 0.67 & 0.26 & 3 & 5 & 7.6 & 3 & 26.52 & 2 & 3 \\
\hline WenzhouT12 & 13.9 & 66.5 & 3 & 0.57 & 0.24 & 7 & 4 & 7.3 & 3 & 22.52 & 3.5 & 3 \\
\hline MaweiT13 & 25.7 & 14.8 & 7 & 0.68 & 0.28 & 5 & 10 & 12.7 & 3 & 16.53 & 2 & 3 \\
\hline XiamenT14 & 23.2 & 105 & 3 & 0.62 & 0.14 & 3 & 4 & 9.2 & 2 & 43.23 & 10 & 2 \\
\hline HuangpuT15 & 25.3 & 73.3 & 3.5 & 0.74 & 0.20 & 8 & 5 & 63.7 & 3 & 42.2 & 3.5 & 2 \\
\hline ShenzhenT16 & 23.0 & 68 & 4 & 0.53 & 0.10 & 2 & 1 & 8.3 & 2 & 64.26 & 10 & 1 \\
\hline ZhanjiangT17 & 13.4 & 11.4 & 3.5 & 0.54 & 0.10 & 18 & 21 & 40.7 & 2 & 32.95 & 7 & 2 \\
\hline
\end{tabular}

For the above 17 evaluation samples, the port resources ecology was evaluated using principal component analysis method adopted $\mathrm{i}$ in the literature [3], and comprehensive evaluation scores of the ports were calculated, the higher the value of port, the higher the level of ecological quality.

\section{Establishment of BP Neural Network Evaluation Model}

\subsection{BP Neural Network Design}

A lot of practice has proved that a 3-layer BP neural network can approximate a nonlinear mapping from input to output, and the reduction of network error is mainly achieved by changing number of nodes in hidden layer instead of increasing number of hidden layers. From the training effect, changing number of hidden layer nodes is easier to observe and adjust. The number of hidden layer nodes is neither too much nor too little, if too much, then the network is easy to transition to fit, which affects the generalization ability, and if too little, then the network learning ability is not strong.

(1) Input layer, the number of nodes $\mathrm{N}$ depends on number of evaluation index. It is 12 in this article.

(2) Output layer, the number of nodes Q depends on classification of evaluation results; it is divided into 5 categories in this article, so $Q$ is 5.

(3) Hidden layer, the number of node R is mainly determined by experimental methods, the following empirical formula is referred in the article, and finally the number of nodes in hidden layer is determined according to the training result. 


$$
r=\sqrt{n+q}+c
$$

Here $\mathrm{n}, \mathrm{q}$ are number of input and output layer neurons, and $c$ is any number between 1 and 10 .

\subsection{BP Neural Network Training}

(1) Due to the inconsistency of units of evaluation index, therefore, when training a sample, it is necessary to perform dimensionless processing on each index, and method is to use the proportional compression to normalize initial data of the sample. Assuming that there are M samples in total, the matrix of initial data is $D=\left(d_{m n}\right)_{M \times N}$, here $d_{m n}$ denotes the initial data of the nth index of the mth sample, the initial data vector of the mth sample is $D_{m}=\left(d_{m 1}, d_{m 2}, \cdots, d_{m N}\right)$, let the normalized initial matrix be $T=\left(t_{m n}\right)_{M \times N}$, here

$$
t_{m n}=\frac{d_{m n}-\min \left(d_{1 n}, d_{2 n}, \cdots, d_{M n}\right)}{\max \left(d_{1 n}, d_{2 n}, \cdots, d_{M n}\right)-\min \left(d_{1 n}, d_{2 n}, \cdots, d_{M n}\right)}
$$

After the mth sample is normalized, the data vector is $T_{m}=\left(t_{m 1}, t_{m 2}, \cdots, t_{m N}\right)$.

(2) Using adaptive Learning Rate Gradient Algorithm, for the ith training, let the learning rate be $\beta_{i}$. The weight of the nth node in input layer to the rth node in hidden layer is $u_{r n, i}$, the threshold at the rth node of hidden layer is $\eta_{r, i}$, the weight of node from the rth node of hidden layer to the qth node of input layer is $w_{q r, i}$, the threshold at the qth node of hidden layer is $\lambda_{q, i}$. When the data vector $\mathrm{Tm}$ is input from input layer, output layer generates an expected value vector based on the evaluation classification result, assume that the expected value vector is: $X_{m}=\left(x_{m 1}, x_{m 2}, \cdots, x_{m Q}\right)$. Let $F_{m, i}=\left(f_{m 1, i}, f_{m 2, i}, \cdots, f_{m R, i}\right)$ is hidden layer output vector, $S_{m, i}=\left(s_{m 1, i}, s_{m 2, i} \cdots, s_{m R, i}\right)$ is output vector of output layer, so output formula of hidden layer node is:

$$
f_{m r, i}=F\left(\sum_{n=1}^{N} u_{r n, i} t_{m n}+\eta_{r, i}\right)
$$

output formula of output layer node is:

$$
s_{m r, i}=S\left(\sum_{r=1}^{R} w_{q r, i} f_{m r, i}+\lambda_{q, i}\right)
$$

error formula is:

$$
E_{i}=\frac{1}{Q M} \sum_{m=1}^{M} \sum_{q=1}^{Q}\left(x_{m q}-s_{m q, i}\right)^{2}
$$

When the $\mathrm{i}+1$ th training is required, the weight adjustment formula is as follows:

Input layer:

$$
\Delta w_{q r, i+1}=\Delta w_{q r, i}+\beta_{i} \frac{\partial E_{i}}{\partial w_{q r, i}}
$$

Hidden layer:

$$
\Delta u_{r n, i+1}=\Delta u_{r n, i}+\beta_{i} \frac{\partial E_{i}}{\partial u_{r n, i}}
$$

The threshold adjustment formula is as follows: 
Input layer:

Hidden layer:

$$
\Delta \lambda_{q, i+1}=\Delta \lambda_{q, i}+\beta_{i} \frac{\partial E_{i}}{\partial \lambda_{q, i}}
$$

$$
\Delta \eta_{r, i+1}=\Delta \eta_{r, i}+\beta_{i} \frac{\partial E_{i}}{\partial \eta_{r, i}}
$$

\subsection{Evaluation Experiment and Analysis}

Use proportional compression formula (2) to normalize the initial data of each index in Table 2 and obtain the normalized initial matrix as follows:

$$
T=\left[\begin{array}{c}
T_{1} \\
\vdots \\
T_{9} \\
\vdots \\
T_{17}
\end{array}\right]=\left[\begin{array}{ccc}
0.383 & \cdots & 0.500 \\
\vdots & \vdots & \vdots \\
0.268 & \cdots & 0.500 \\
\vdots & \vdots & \vdots \\
0.079 & \cdots & 1
\end{array}\right]
$$

The BP neural network model adopts a $12 \times 7 \times 5$ structure, network training uses vector data corresponding to $\mathrm{T} 1$ to $\mathrm{T} 10$ in the matrix as a training sample to train the BP network: the vector data corresponding to T1-T10 is used as the input of network, and the expected value vector of sample classification is used as the output, the expected value vector is shown in Table 3 . Design the maximum number of learning times is 20,000 , learning rate is 0.005 , learning target square error is 0.1 and accuracy of error convergence is $10-3$. When training step is 2835 , training error reaches $1 \times 10$ 3 , and it meets original design requirements.

Table.3 Expectation vectors for categories of samples

\begin{tabular}{|c|c|c|c|c|c|}
\hline & excellent & good & medium & poor & worst \\
\hline$x_{m 1}$ & 1 & 0 & 0 & 0 & 0 \\
\hline$x_{m 2}$ & 0 & 1 & 0 & 0 & 0 \\
\hline$x_{m 3}$ & 0 & 0 & 1 & 0 & 0 \\
\hline$x_{m 4}$ & 0 & 0 & 0 & 1 & 0 \\
\hline$x_{m 5}$ & 0 & 0 & 0 & 0 & 1 \\
\hline
\end{tabular}

Using Matlab 7.0 programming, BP neural network is simulated, and input vector data corresponding to T1-T10 in the matrix are inputted into the trained neural network, then mean square error value of output vector and expected value vector corresponding to each sample category is calculated, the sample category with the smallest mean square error is desired sample classification result. Compared the classification results to be obtained with comprehensive evaluation results of principal component analysis method above-described. The results are shown in Table 4.

From Table 4, it can be seen that among the 17 valid samples, there are 15 consistent evaluation results, and only three sample evaluation results in inconsistent, which are Lianyungang, Shanghai, and Wenzhou, it shows that the established BP neural network evaluation model is accurate and effective for evaluation of ecological quality of port resources. 
Table.4 Evaluation results

\begin{tabular}{|c|c|c|c|c|c|}
\hline port & $\begin{array}{c}\text { BP } \\
\text { network }\end{array}$ & $\begin{array}{c}\text { principal component } \\
\text { analysis }\end{array}$ & port & $\begin{array}{c}\text { BP } \\
\text { network }\end{array}$ & $\begin{array}{c}\text { principal component } \\
\text { analysis }\end{array}$ \\
\hline Dalian & worst & -0.113 & Beilun & good & 0.081 \\
\hline Qinhuangdao & medium & 0.042 & Taizhou & poor & -0.031 \\
\hline Tianjin & excellent & 0.156 & Wenzhou & medium & 0.057 \\
\hline Yantai & worst & -0.136 & Mawei & medium & 0.045 \\
\hline Qingdao & worst & -0.127 & Xiamen & excellent & 0.144 \\
\hline Rizhao & good & 0.783 & Huangpu & good & 0.083 \\
\hline Lianyungang & poor & 0.018 & Shenzhen & excellent & 0.168 \\
\hline Shanghai & good & 0.107 & Zhanjiang & excellent & 0.151 \\
\hline Zhangjiagang & medium & 0.034 & & & \\
\hline
\end{tabular}

\section{Conclusion}

(1)The BP neural network evaluation model was used to evaluate 17 valid samples and results were basically consistent with the principal component analysis method, which proved that the evaluation method was effective.

(2) The principal component analysis method is a simplified data set technology, in the evaluation process; the original index information will be lost, which resulting in lack of accuracy of evaluation results. The BP neural network evaluation method is a full-information artificial intelligence evaluation method with high evaluation accuracy, and the evaluation model is further improved with the increase of training sample data.

\section{References}

[1]. Clark X.,Dollar D.,Micco A. Port efficiency, maritime transport costs, and bilateral trade [J]. Journal of Development Economics, 2004(75): 417-450.

[2]. Liu, Z. The comparative performance of public and private Enterprises: The Case of British Ports [J]: Journal of Transport Economics and Policy, 1995, 29(3): 263-274.

[3]. ROLL Y, HAYUTH Y. port performance comparison applying data envelopment analysis[J]: Maritime Policy and Management, 1993, 20(2): 153-161.

[4]. XIE Feng, MA Zhimin, LUAN Weidong. Quality evaluation of expressway pavement based on fuzzy neural networks[J]. Journal of Southwest Jiaotong University, 2013,48(1): 160-164.

[5]. ZHANG Jianping, HU Minghua, WU Zhenya, et al. An improved integrated evaluation method on operation performance of air traffic control based on BP. 